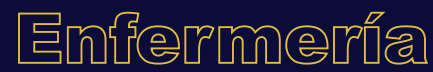

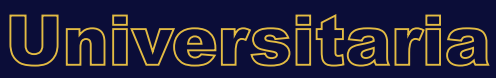

\section{Factores emocionales asociados al automanejo en personas con diagnóstico de cáncer}

\section{Emotional factors associated to self-management in persons diagnosed with cancer}

\section{Fatores emocionais associados à autogestão em pessoas com diagnóstico de câncer}

\section{T. Gutiérrez-Gómez ${ }^{\text {a1 }}$, M.I. Peñarrieta-de Cordova ${ }^{\text {b1 }}$, D.J. Malibrán-Luque ${ }^{\mathrm{c2} *}$, M.S. Piñones-Martínez ${ }^{\mathrm{d} 1}$, M. Cosme-Mendoza ${ }^{\mathrm{e}}$, N. Gaspar Meza-de Nalvarte ${ }^{\mathrm{f}_{3}}$}

ORCID

a 0000-0001-5522-4237

d $\underline{0000-0002-1013-7700}$

${ }^{\mathrm{b}}$ 0000-0002-9293-4024

e oooo-0003-2279-2873

c0000-0003-3380-1528

f Oooo-0001-7596-8719

${ }^{1}$ Universidad Autónoma de Tamaulipas, Facultad de Enfermería, Tampico, Tamaulipas, México

${ }^{2}$ Universidad Autónoma de Tamaulipas, Facultad de Medicina Dr. Alberto Romo Caballero, Tampico, Tamaulipas, México

${ }^{3}$ Instituto Nacional de Enfermedades Neoplásicas, Área de Investigación del Departamento de Enfermería, Lima, Perú

Recibido: 13 agosto 2020

Aceptado: 25 mayo 2021

RESUMEN

Introducción: Las personas con diagnóstico de cáncer y que reciben tratamiento manifiestan factores estresantes, físicos, emocionales y sociales, que inciden en la salud mental del paciente, por lo tanto, se reconoce la importancia de las prácticas de automanejo para aliviar la carga de estos factores.

* Autor para correspondencia. Correo electrónico: dmalibran@docentes.uat.edu.mx https://doi.org/10.22201/eneo.23958421e.2021.2.957

1665-7063/@ 2021 Universidad Nacional Autónoma de México, Escuela Nacional de Enfermería y Obstetricia. Este es un artículo Open Access bajo la licencia CC BY-NC-ND (http://creativecommons.org/licenses/by-nc-nd/4.o/). 
Objetivo: Identificar factores emocionales que puedan ser útiles para predecir la práctica de automanejo.

Método: Diseño transversal, predictivo, muestreo no probabilístico intencional conformado por 160 personas con diagnóstico de cáncer. Se emplearon los instrumentos: Partners in Health (PIH), Self-rated Health, Escala de depresión del Cuestionario de Salud del Paciente (PHO-8P), Escala Visual Análoga para valorar el estrés e insomnio. Se aplicó el modelo de regresión lineal múltiple; se calculó el efecto de la diferencia y la potencia estadística utilizando el método de escalonado.

Resultados: Se identificaron dos variables predictoras con significancia estadística ( $p<.05)$ : problemas para dormir $\beta=-1.310$ y trastorno depresivo $\beta=-.440$, con una varianza del $10 \%$, prueba de Durbin-Watson 1,658, tamaño del efecto 0.15 y una potencia de 0.95 .

Discusión y Conclusiones: Las personas con cáncer presentan una práctica de automanejo deficiente. Se identificaron factores emocionales que interfieren en este comportamiento: estrés, trastorno depresivo, y problemas para dormir; los dos últimos factores pueden influir en el automanejo. Se sugiere seguir investigando y profundizar el estudio con el fin de identificar otras barreras asociadas al automanejo, sus diferenciaciones según perfiles sociodemográficos y estadio de la enfermedad.

Palabras clave: Autocuidado; automanejo; cáncer; factores emocionales; Perú.

\section{ABSTRACT}

Introduction: Persons diagnosed with cancer and who are under treatment suffer from physical, emotional, and social stress which in turn has an impact on their mental health. Therefore, it is important to explore the self-management practices aimed at relieving the burden of the related stress associated factors.

Method: This is a transversal and predictive study with intentional and non probabilistic sampling on 160 persons diagnosed with cancer. The Partners in Health (PIH), Self-rated Health, Depresion of the PHO-8, as well as the Analogous Visual Scales were used. A multiple linear regression model, the effect of the difference, and the statistical power using the stepwise method were all calculated.

Results: Two statistically significant predicting variables ( $\mathrm{p}<.05)$ were identified: sleep problems $\beta=-1.310$ and depressive disorder $\beta=-.440$, with a variance of $10 \%$, Durbin-Watson test of 1,658, effect size of 0.15 , and a power of 0.95 .

Discussion and conclusion: Persons with cancer usually demonstrate deficient self-management practices. Emotional factors which drive these behaviors were identified: stress, depressive disorder, and sleep problems. It is suggested to conduct further research in order to identify other social and demographic barriers related to the self-management of cancer. Keywords: Self-care; self-management; cancer; emotional factors; Peru.

\section{RESUMO}

Introdução: As pessoas diagnosticadas com câncer e que recebem tratamento manifestam estresse físico, emocional e social, que afetam a saúde mental do paciente, portanto, é reconhecida a importância das práticas de autogestão para aliviar o peso destes fatores.

Objetivo: Identificar fatores emocionais que possam ser úteis para prever a prática de autogestão. 
Método: Amostra transversal, preditivo, não probabilístico intencional composta por 160 personas com diagnóstico de câncer. Os instrumentos utilizados foram: Partners in Health (PIH), Self-rated Health, Escala de depressão do Questionário de Saúde do Paciente (PHO-8), Escala Visual Analógica para avaliar o estresse e insônia. O modelo de regressão linear múltipla foi aplicado; calculou-se o efeito da diferença e o poder estatístico usando o método por etapas.

Resultados: Duas variáveis preditivas com significância estatística ( $\mathrm{p}<.05)$ foram identificadas: problemas de sono $\beta=-1.310$ e transtorno depressivo $\beta=-.440$, com una variância de $10 \%$, teste de Durbin-Watson 1,658, tamanho de efeito o.15 e uma potência de 0.95 .

Discussão e Conclusões: As pessoas com câncer apresentam uma prática de autogestão deficiente. Foram identificados fatores emocionais que interferem neste comportamento: estresse, transtorno depressivo, e problemas de sono; os dois últimos fatores que podem influenciar a autogestão. Sugere-se continuar pesquisando e aprofundar o estudo a fim de identificar outras barreiras associadas à autogestão, suas diferenciações segundo os perfis sociodemográficos e o estádio da doença.

Palavras chave: Autocuidado; autogestão; câncer; fatores emocionais; Peru.

\section{INTRODUCCIÓN}

El cáncer es un problema significativo de salud pública a nivel mundial dada la carga de enfermedad y mortalidad que produce ${ }^{1}$. Para el año 2018, GLOBOCAN estimó 18.1 millones de casos nuevos y 9.6 millones de muertes por esta enfermedad; se calcula que la prevalencia en los últimos 5 años se estima en 15 millones de personas ${ }^{1,2}$.

Actualmente, el Perú se encuentra en una transición demográfica que tiene impacto en la incidencia y prevalencia del cáncer ${ }^{3}$. De acuerdo con estimados de la OMS, durante el 2015, en diferentes países, esta fue la primera o segunda causa de muerte en personas menores de 70 años². Para el país andino las neoplasias malignas, se ubicaron en segunda posición, superadas solo por las enfermedades infecciosas y parasitarias 4 .

La mortalidad prematura es definida como el porcentaje de defunciones que se producen por una enfermedad no transmisible entre los 30 y 69 años. En el Perú, la muerte prematura por cáncer para el año 2016 presentó una reducción del 5.1\% en comparación con el año 20064. De acuerdo con el estudio internacional CONCORD-3, el cual registró datos de 37.5 millones de pacientes diagnosticados con este padecimiento en 71 países durante un periodo de 15 años (2000-2014), la tasa de supervivencia de cinco años después del diagnóstico fue alta en Estados Unidos, Canadá, Australia, Nueva Zelanda y en los países nórdicos5.

En Perú, las neoplasias con el porcentaje más alto de supervivencia estimada a cinco años, según The Mortality-Incidence Ratio complement (1-MIR), son tiroides (86.7\%), mama (68\%), próstata (63.8\%) y cáncer de cuello uterino (58.5\%). Estos resultados se obtuvieron utilizando el complemento MIR calculado a partir de datos del Registro de Cáncer de Lima Metropolitana ${ }^{6}$.

El incremento en incidencia y prevalencia trajo consigo el aumento en el número de sobrevivientes del cáncer-7, lo que significa vivir a largo plazo con efectos de la enfermedad y su tratamiento, por ejemplo, manifestar fatiga crónica, depresión, ansiedad, cambios cognitivos y nuevos problemas de salud, como: segundas neoplasias, enfermedades cardiovasculares y trastornos endocrinos. También, puede presentarse la interrupción en las actividades diarias, dolor físico, disminución de 
energía, cambios en la apariencia física, limitaciones en la capacidad funcional, relaciones sociales alteradas, confrontación con la mortalidad, problemas existenciales y cambios en el sentido de su futuro 8 .

El cáncer junto con el tratamiento se acompañan de factores estresantes físicos, emocionales, sociales, ocupacionales y financieros, así como de numerosos síntomas fisiológicos, por ejemplo: fatiga, trastornos del sueño, dolor, náuseas y vómitos, deterioro del funcionamiento sexual y problemas cognitivos, entre otros 9 ; además del aumento en la ansiedad y síntomas depresivos ${ }^{10}$. La ansiedad en torno al diagnóstico de cáncer puede provocar trastornos del sueño, lo que ocasiona mayor riesgo de presentar depresión ${ }^{11}$, asociado al fuerte impacto en la salud mental entre las personas con cáncer ${ }^{12}$.

Los modelos actuales de atención, enfocados principalmente en la detección de recurrencias, no abordan de manera adecuada las necesidades integrales de los sobrevivientes, quienes requieren de atención médica y de apoyo continuo, además de una interacción frecuente con múltiples proveedores de atención médica ${ }^{13-15}$. En las últimas dos décadas se ha incrementado el reconocimiento a los programas de automanejo, al identificar que estos pueden representar una estrategia efectiva para garantizar que se aborden las necesidades de salud física y psicológica a largo plazo de los sobrevivientes ${ }^{13-16}$. Lo que significará un cambio en el enfoque tradicional del manejo de la cronicidad basado en el proveedor, a un nuevo paradigma en el que las personas con enfermedad crónica juegan un rol proactivo de su propio cuidado en conjunto con los proveedores de salud para el control de su condición.

Nos referimos al automanejo, como las tareas que los individuos deben emprender para vivir bien con una o más condiciones crónicas ${ }^{17}$; las cuales incluyen desarrollar la confianza para lidiar con la atención médica, el manejo de roles y el cuidado emocional de sus condiciones ${ }^{18,19}$. El autocuidado representa un nuevo modelo de atención centrado en el paciente $e^{20}$, donde uno de los objetivos centrales es promover el automanejo de la condición crónica. Este es considerado como una parte esencial en el cuidado de la persona que vive en condiciones crónicas, dando origen a muchos programas que promueven dicho comportamiento ${ }^{21}$.

La participación de los pacientes con cáncer en las prácticas de automanejo se ha convertido en una prioridad de la reforma para la atención de esta enfermedad ${ }^{22}$. Se reconoce la importancia de la participación activa del paciente con prácticas de automanejo para aliviar la carga de los efectos secundarios, tanto físicos como psicológicos ${ }^{23-25}$. Las categorías de automanejo más reportadas corresponden al tipo de dieta, nutrición, estilo de vida que incluye acciones de autocontrol (por ejemplo: el consumo de fibra para el estreñimiento o la lectura como recurso para el insomnio). Las categorías menos estudiadas son: consumo de hierbas, vitaminas y terapia complementaria ${ }^{26}$; la comunicación con familiares, amigos o proveedores de la salud; el manejo de los síntomas, adhesión y toma de decisión respecto al tratamiento; aprender sobre el cáncer, así como y mantener la calidad de vida ${ }^{26-29}$.

En Latinoamérica aún no se cuenta con los suficientes conocimientos sobre este comportamiento en personas con cáncer. Un estudio exploratorio con población peruana demostró que las personas con cáncer presentan un automanejo deficiente, sobre todo en el nivel de conocimiento y el manejo del impacto de la enfermedad en los aspectos físicos, emocionales y sociales ${ }^{30}$. Peñarrieta-de Córdova et al. ${ }^{31}$ mencionan que este concepto combina aspectos médicos (biológicos), psicológicos y sociales que podrían aplicarse a cualquier condición crónica. En este sentido, el automanejo [...] implica participar en actividades que protegen y promueven la salud, el monitoreo y control de síntomas 
y signos de enfermedad, así como el impacto de la enfermedad en el funcionamiento, las emociones, relaciones interpersonales y adhesión al régimen del tratamiento 29.

Además de las prácticas de automanejo, existen factores asociados con este comportamiento en los pacientes con cáncer. Dichas prácticas varían entre pacientes ya que responden a características demográficas y de personalidad, experiencias de enfermedades y necesidades, apoyo social y estilo de afrontamiento ${ }^{22,33}$. En diversas investigaciones se han identificado barreras de diferente índole, ya sea emocionales, derivadas de los síntomas adversos; estructurales, relacionadas con el acceso a servicios de salud y de autoevaluación ${ }^{34,35}$. Aunado a esto, la falta de comprensión del cáncer y su trayectoria ${ }^{28}$; la edad, el sexo, así como el nivel económico se asocian con las prácticas de automanejo ${ }^{29,36}$.

Actualmente, lo que se conoce en población latinoamericana de estos factores asociados al automanejo es aún incipiente. Por consiguiente, el presente estudio tiene como objetivo identificar factores emocionales que puedan ser útiles para predecir la práctica de automanejo y así facilitar la detección temprana y las intervenciones a medida, lo que permitirá optimizar la salud de los sobrevivientes de cáncer vulnerables.

Los profesionales de enfermería desempeñan un papel importante en el seguimiento de la evolución del paciente, el asesoramiento, la educación sanitaria y la orientación sobre el automanejo durante la atención a largo plazo de las personas con cáncer. Cuando el personal médico es limitado, el de enfermería debe priorizar a los pacientes más vulnerables. Al distinguir entre los diferentes niveles en la práctica de automanejo, así como los factores o barreras emocionales para promover este comportamiento, el personal de enfermería reconoce con prontitud a las personas vulnerables, lo que podría permitir brindar una atención más personalizada. En este sentido, se busca maximizar el apoyo a los pacientes vulnerables y minimizar la atención innecesaria de aquellos con un nivel adecuado de automanejo para conducir a intervenciones más rentables, que incida en un mejor cuidado personal y calidad de vida en los sobrevivientes de cáncer.

\section{MÉTODOS}

\section{Diseño del estudio}

El estudio fue un diseño transversal, predictivo. Esta investigación forma parte de un proyecto de matriz binacional entre la Red de automanejo de enfermedades crónicas y el Departamento de Enfermería del Instituto Nacional de Enfermedades Neoplásicas de Lima, Perú. La investigación se llevó a cabo durante el periodo 2016-2017.

\section{Contexto (población)}

La población de estudio fueron personas con diagnóstico de cáncer, usuarios de servicios de consulta externa y quimioterapia del Instituto Nacional de Enfermedades Neoplásicas de Lima, Perú.

\section{Mediciones}

Se realizó un muestreo no probabilístico intencional, respetando las exigencias de participación del programa Tomando Control de su Salud de la Universidad de Stanford ${ }^{37}$, aplicado por dos personas previamente certificadas como Líderes; cada grupo participante no debía ser menor a 12 ni mayor a 16, se consideró el total de personas con alguna condición crónica registradas en el Instituto Nacional de Enfermedades Neoplásicas. Se trabajó con la base de datos de las mediciones antes de la implementación del programa realizado en 2016-17. La muestra quedó conformada por 160 personas con diagnóstico de cáncer. 


\section{Criterios de selección y exclusión}

Los criterios de inclusión fueron: ser usuarios ambulatorios (consulta externa, quimioterapia) mayores de 18 años con diagnóstico de cáncer sin importar el tipo de neoplasia o tratamiento que estuviera recibiendo, dado que no fue de interés para el objetivo inicial de la investigación. Los criterios de exclusión establecidos son: usuarias con diagnóstico de cáncer embarazadas, que presentaran problemas cognitivos (orientado en tiempo, persona y lugar) y no estar recibiendo actualmente un tratamiento agudo frente al cáncer o estar en etapa terminal.

\section{Instrumentos de medición}

\section{a) Escala de Automanejo de enfermedades crónicas}

Se midió el automanejo a través del instrumento Partners in Health (PIH) diseñado por la Universidad de Flinders, Australia ${ }^{38}$. El cuestionario consta de 12 reactivos con una escala tipo Likert, puntajes que van del 0 al 8, donde 0 indica automanejo bajo y 8 corresponde a automanejo alto; fue validado en población peruana con un Alpha de Cronbach de 0.72. El PIH mide tres dimensiones: conocimiento (preguntas 1 y 2); adherencia al cuidado (preguntas 3-8 y 12) y; manejo del impacto psicológico, físico y social de la condición crónica (preguntas 9-11). La fiabilidad estadística por cada dimensión fue 0.78 para conocimiento; 0.96 para la adherencia a cuidados médicos y 0.70 para el manejo de salud ${ }^{39}$.

La interpretación de los resultados es a mayor puntaje mejor automanejo. La sumatoria global va en rangos de 0 a 96; conocimiento de 0-16; cuidados médicos de 0-56 y; manejo del impacto físico, emocional y social de 0-24. Este instrumento evalúa el comportamiento desde la perspectiva del proveedor de salud al ser él quien realiza las preguntas y da un puntaje según su criterio; también puede ser autoadministrado a la persona con condición crónica, siendo ella la que da el puntaje a la pregunta. En este estudio se recoge la perspectiva de la persona crónica, siendo ella la que determina el puntaje que consideraba se ajustaba a cada pregunta ${ }^{40}$.

\section{b) Factores emocionales}

Se consideraron las siguientes variables:

- Autopercepción de salud

Para la medición de la autopercepción de salud se empleó el test Self-rated Health (SRH), que consta de una sola pregunta con cinco opciones de respuesta: 1) excelente, 2) muy buena, 3) buena, 4) regular, 5) mala. Para efectos del análisis de regresión se dicotomizó esta variable en dos categorías: 1) excelente, muy buena y buena, 2) regular y mala. El coeficiente de correlación test-retest es de $0.92^{41,42}$. Este instrumento fue validado en una muestra de 250 participantes que tenían alguna enfermedad crónica, derechohabientes de los Centros de salud, y se obtuvo la confiabilidad test-retest con un .685 (<.001) 43 .

\section{- Trastorno depresivo}

Se aplicó la escala de depresión del Cuestionario de Salud del Paciente (PHQ-8), el cual consta de 8 ítems en forma de pregunta, cada uno de estos tiene cuatro alternativas de respuesta, evalúa la sintomatología experimentada en las últimas dos semanas. Cada ítem tiene un valor que va de $0 \mathrm{a}$ 3 puntos en función de la alternativa elegida y tras sumar directamente la puntuación de cada uno se obtiene una puntuación global que va de 0 a 24 puntos totales. La validación mostró consistencia 
interna de 0.89 , con una sensibilidad de 88\% y mismo porcentaje de especificidad para la depresión mayor ${ }^{44}$. La validación en población peruana tuvo Alpha de Cronbach de 0.78 y test-retest de $0.85^{45}$.

- Estrés e insomnio

Ambas variables se miden a través de una escala visual análoga de una sola pregunta, la cual consta de diez barras de diferentes alturas e intensidades de sombreado, esto facilita la respuesta por parte del participante del estudio. La escala numérica análoga del estrés (Escala visual analógica de estrés) tiene resultados de validez aceptables en población latina ${ }^{46,47}$. La escala numérica visual de problemas para dormir ha sido contrastada con otros test similares demostrando resultados semejantes ${ }^{48,49}$. Ambas escalas son utilizadas en la evaluación del Programa de automanejo en crónicos de la Universidad de Stanford ${ }^{41,5}$. Se utilizó una pregunta adicional sobre el número de problemas de salud mental presentados durante el mes pasado, derivados por la condición crónica del participante (pregunta también utilizada por el Programa de Stanford en su evaluación)41,42.

- Datos sociodemográficos

Las variables analizadas incluyen edad, número de años, sexo, estado civil (casado y soltero), procedencia (Lima-fuera de Lima), estudios (básicos hasta secundaria y superiores) y número de miembros de familia.

- Datos de salud

Para explorar aspectos de salud se consideraron dos variables: el tiempo transcurrido desde el diagnóstico de la enfermedad hasta la incorporación al proyecto de investigación y la presencia de otra enfermedad adicional.

Las variables sociodemográficas y de salud fueron consideradas como variables de ajuste al modelo de regresión aplicado.

\section{Aplicación de la encuesta}

La encuesta fue aplicada por estudiantes del último año de Enfermería, previamente capacitadas. La duración de la aplicación fue de 30 minutos, se llevó acabo en los mismos servicios de salud en un ambiente acondicionado sin ruido y con privacidad. No se presentaron problemas en su aplicación.

\section{Análisis estadístico}

Para el procesamiento de datos se utilizó el paquete estadístico SPSS versión 26, mostrando la variable dependiente (Automanejo) normalidad (prueba de Kolmogorov Smirnov p>.05). Se calculó la correlación entre el automanejo y las variables independientes (factores emocionales) mediante la técnica de Correlación de Pearson. Se seleccionaron los factores que dieron significancia estadística $\mathrm{p} \leq 0.05$. Dichas variables se incluyeron en el modelo de regresión lineal múltiple, entre ellas las variables de ajuste (edad, sexo, años con la enfermedad, presencia de comorbilidad); con estas se generaron dos modelos a través del método de escalonado de pasos. De estos modelos se calculó el tamaño del efecto (d) con el programa $G^{*}$ power, con la finalidad de predecir el automanejo y si esta predicción es relevante a través de los puntos de corte (pequeño .02, mediano .15 y grande .35). De igual forma, se calculó la potencia estadística (1- $\beta$ ), esto es la capacidad de generalizar los resultados (mínimo .80) $)^{51}$. 


\section{ASPECTOS ÉTICOS}

Se solicitó el consentimiento informado a cada participante, a quienes se les explicó los objetivos del estudio y garantizando la inocuidad durante el desarrollo de la investigación. Estos tenían la libertad de abandonar la investigación cuando lo consideraran sin ningún compromiso. Se contó además con la aprobación del Comité de Ética de la Facultad de Enfermería donde está la sede del proyecto (Dictamen: Número de registro 004-2019) y de las instituciones participantes (Ministerio de Salud e Instituto Nacional de Enfermedades Neoplásicas de Lima, Perú).

\section{LIMITACIONES DEL ESTUDIO}

Al ser una muestra por conveniencia los resultados no pueden generalizarse. Cabe señalar que en este análisis no se consideraron variables referidas en otros estudios como factor emocional (entre ellas la autoeficacia) y variables confusoras (tipo cáncer y tratamiento). Sin embargo, consideramos que, a pesar de estas limitaciones, los resultados son válidos y confiables, permitiendo conseguir el objetivo del presente estudio.

\section{RESULTADOS}

La muestra estuvo conformada en mayor medida por mujeres adultas, con una media de 56 años, poco más de la mitad son casados, $40 \%$ residen en otras ciudades del país, la cuarta parte presenta una enfermedad adicional al cáncer. Se tiene una media de 6 años desde el diagnóstico de la enfermedad hasta la incorporación en el proyecto de investigación (Tabla 1).

Tabla 1. Aspectos sociodemográficos y condiciones de salud

\begin{tabular}{lcc} 
Aspectos sociodemográficos & F & $\%$ \\
\hline Edad en años & $(\mathrm{n}: 160)$ & $(100)$ \\
\hline Educación (años de estudio) & $\bar{x}: 55.97$ & Rangos: Min: 18 Max:90 \\
\hline Sexo & $\bar{x}: 9.7$ & Rangos: Min: 2 Max:20 \\
Varón & 56 & 35.0 \\
\hline Mujer & 104 & 65.0 \\
\hline Estado Civil & & \\
Casado & 101 & 63.1 \\
\hline Viudo & 20 & 12.5 \\
\hline Divorciado & 8 & 5.0 \\
\hline Soltero & 31 & 19.4 \\
\hline Tipo de Familia & & \\
\hline Nuclear & 90 & 56.3 \\
\hline Otros & 70 & 43.7 \\
\hline Procedencia & & \\
\hline Lima & 97 & 60.6 \\
\hline Costa & 20 & 12.5 \\
\hline
\end{tabular}




\begin{tabular}{lcc}
\hline Sierra & 33 & 20.6 \\
\hline Selva & 10 & 6.3 \\
\hline Tipo de seguro & 150 & 93.8 \\
\hline Subsidio & 10 & 6.2 \\
\hline Privado & & \\
\hline Comorbilidad & 125 & 78.1 \\
\hline Sí & 35 & 21.9 \\
\hline No & $\bar{x}: 4.9$ & Rangos: Min: 2 Max: 11 \\
\hline No. de miembros en la familia & $\bar{x}: 5.9$ & Rangos: Min:1 Max:47 \\
\hline No. de años de la enfermedad & & \\
\hline
\end{tabular}

Respecto a los factores emocionales $65 \%$ refiere algún nivel de trastorno depresivo, la mitad de la población presenta una media de 5 puntos en un rango de o a 10 en problemas para dormir y estrés, poco más de la mitad de la muestra reportó una percepción de su salud regular (Tabla 2).

\begin{tabular}{lcc}
\multicolumn{4}{c}{ Tabla 2. Descripción de los factores emocionales } \\
Variables & Media & \\
\hline Problemas para dormir la semana pasada (1-10) & $\bar{x}: 4.91$ & \\
\hline Estrés (1-10) & $\bar{x}: 5.86$ & \\
\hline \multicolumn{1}{c}{ F } & & \\
\hline Trastornos depresivos & 56 & 35.0 \\
\hline Sin síntomas & 52 & 32.5 \\
\hline Síntomas leves & 27 & 16.9 \\
\hline Síntomas moderados & 22 & 13.8 \\
\hline Síntomas moderadamente severos & 3 & 1.9 \\
\hline Síntomas severos & & \\
\hline Percepción de su salud & 3 & 1.9 \\
\hline Muy buena & 57 & 35.6 \\
\hline Buena & 93 & 58.1 \\
\hline Regular & 7 & 4.4 \\
\hline Mala & & \\
\hline
\end{tabular}

Con relación al automanejo, en el índice general se obtuvo una media de 73.12 puntos en un rango de o-96, los resultados muestran en las tres dimensiones puntajes por debajo de la medición ideal (Tabla 3). 


\section{Tabla 3. Caracteristicas del automanejo en personas con cáncer}

\begin{tabular}{|c|c|c|}
\hline Dimensión & Media & Preguntas \\
\hline \multirow{2}{*}{ Conocimiento $(0-16)$} & \multirow{2}{*}{10.99} & 1. Conocimiento de su estado de salud (0-8) \\
\hline & & 2. Conocimiento de su enfermedad (0-8) \\
\hline \multirow{7}{*}{$\begin{array}{l}\text { Adherencia a cuidados } \\
\text { médicos }(0-56)\end{array}$} & \multirow{7}{*}{43.58} & 3. Toma las medicinas (0-8) \\
\hline & & $\begin{array}{l}\text { 4. Comparte las decisiones con proveedores de salud } \\
(0-8)\end{array}$ \\
\hline & & 5. Solicitar los servicios (0-8) \\
\hline & & 6. Asiste a las citas (0-8) \\
\hline & & 7. Signos de alerta (0-8) \\
\hline & & 8. Toma medidas a señales de advertencia (0-8) \\
\hline & & 12. Estilo de vida saludable (0-8) \\
\hline \multirow{3}{*}{$\begin{array}{l}\text { Automanejo Impacto } \\
\text { físico, emocional y social } \\
(0-24)\end{array}$} & \multirow{3}{*}{18.53} & 9. Manejo de efectos secundarios físicos (0-8) \\
\hline & & 10. Manejo efecto emocional $(0-8)$ \\
\hline & & 11. Manejo efecto social (0-8) \\
\hline Índice general (0-96) & 73.12 & (Las 12 preguntas) \\
\hline
\end{tabular}

Los resultados de correlación entre los factores emocionales y el automanejo, en su mayoría presentaron una correlación significativa ( $\mathrm{p}<.05)$ : trastorno depresivo, estrés, problemas para dormir; a excepción de la percepción de su salud como se muestra en la tabla 4.

\section{Tabla 4. Correlación entre los factores emocionales y automanejo}

\begin{tabular}{lcc} 
Variables & Corr & Sig \\
\hline Problemas para dormir la semana pasada & -.264 & .001 \\
\hline Trastorno depresivo & -253 & .001 \\
\hline Estrés & -.225 & .004 \\
\hline Percepción de su salud & .078 & .338 \\
\hline
\end{tabular}

En el modelo se incluyeron las variables que mostraron significancia estadística en la correlación, así como las variables sociodemográficas y de salud que son variables de ajuste. Después de eliminar las variables no significativas para el modelo, los resultados de la regresión muestran dos de ellos: en el modelo uno solamente se identifica a la variable: problemas para dormir con significancia estadística ( $p<.05$ ), con el 6\% de la varianza explicada, mostrando un tamaño del efecto .07 y una potencia de $\beta$.93. En el segundo modelo sobresalen dos variables: problemas para dormir y trastorno depresivo, la beta correspondiente a la primera variable que es -1.310 , la beta para la segunda es -.440 con significancia estadística ( $\mathrm{p}<.05$ ). Este valor negativo nos indica que por cada unidad de cambio en las betas al aumentar uno disminuye la otra variable; es decir, al aumentar problemas para dormir y trastorno depresivo disminuye el automanejo, explicando una varianza del 10\%. El resultado de la 
prueba de Durbin-Watson muestra un ajuste del modelo 1.658 (valores entre 1-3) mostrando un tamaño del efecto 0.15 y una potencia de 0.95 (Tabla 5).

\section{Tabla 5. Regresión de las variables predictoras en el automanejo}

\begin{tabular}{lccccc} 
Modelos & R2 & B & p & $1-\beta$ & $f$ \\
Modelo 1 & .064 & & & .93 & .07 \\
\hline Problemas para dormir & & -.284 & .001 & & \\
Modelo 2 & .096 & & & .95 & .15 \\
\hline Problemas para dormir & & -.196 & .018 & & \\
\hline Trastorno depresivo & & -.177 & .033 & & \\
\hline
\end{tabular}

\section{DISCUSIÓN}

Los resultados del presente estudio nos permiten corroborar la presencia de problemas emocionales en personas con cáncer tales como trastorno depresivo, estrés, autopercepción regular de la salud y problemas para dormir ${ }^{10-12}$; además de alteraciones presentes en los sobrevivientes de esta enfermedad, por ejemplo, ansiedad y autoeficacia disminuida, resultados que son similares a otras investigaciones ${ }^{11,12}$. Diversos estudios también identificaron problemas físicos y sociales ${ }^{9-11}$ que ameritarían ser investigados en población peruana y latinoamericana con el fin de evidenciar la importancia del correcto automanejo en estos pacientes, que les permita lidiar día a día con el manejo de esta enfermedad y garantizar una mejor calidad de vida.

Se identificó una práctica de automanejo deficiente, aspectos como: tener conocimientos necesarios de la condición de salud y enfermedad; el manejo del impacto físico, emocional y social ante la enfermedad; una participación activa con el proveedor de salud; monitoreo de los síntomas y adecuado estilo de vida fueron resultados similares a los reportados en otro estudio con población peruana ${ }^{30}$. En ese sentido, se evidencia la necesidad que tiene el cambio del enfoque tradicional de manejo de la cronicidad a un nuevo paradigma en el que las personas con enfermedad crónica tengan un rol proactivo de su propio cuidado (automanejo) ${ }^{20,22}$. Esto último se confirma con la presencia cada vez mayor de programas que promueven el automanejo en personas con neoplasias, con resultados muy alentadores en la calidad de vida de sus participantes 23-25. $^{2}$

Con esta investigación se busca contribuir a esta área, identificando factores emocionales que puedan ser útiles para predecir la práctica de automanejo. Los resultados muestran que de los factores seleccionados: problemas para dormir, estrés, percepción de su salud y trastorno depresivo tienen relación significativa entre tres de ellos, a excepción de la percepción de su salud. Sin embargo, de estos tres, dos son los que muestran impacto predictivo en el automanejo: problemas para dormir y trastorno depresivo. Si existen muchos problemas para dormir y un elevado estado depresivo se puede predecir un automanejo bajo, resultados similares con otros estudios en los que se ha identificado algunas barreras psicológicas en el automanejo ${ }^{32-34}$ como son: el miedo, incertidumbre, falta de control, entre otras. 
Estos resultados permiten al personal de salud y en especial al personal de enfermería, quienes desempeñan un rol muy importante en el seguimiento y educación de estos pacientes, identificar oportunamente la presencia de estos dos factores emocionales con el fin de realizar intervenciones oportunas y personalizadas para promover el automanejo.

\section{CONCLUSIONES}

Los hallazgos nos permiten afirmar que las personas con cáncer presentan una práctica de automanejo deficiente; se identificaron factores emocionales que interfieren en este comportamiento: estrés, trastorno depresivo y problemas para dormir, los dos últimos son predictores del automanejo. Al ser una de las primeras investigaciones realizadas con población peruana, es muy importante seguir abordando en este tema, sobre todo profundizar el estudio para identificar otras barreras asociadas al automanejo, sus diferenciaciones según perfiles sociodemográficos y estadio de la enfermedad.

\section{RESPONSABILIDADES ÉTICAS}

Protección de personas y animales. Los autores declaran que han obtenido el consentimiento informado de los sujetos referidos en el estudio. No se realizaron experimento en personas ni animales.

Confidencialidad. Los datos recabados fueron protegidos conforme al reglamento de la Ley General de Salud en Materia de Investigación para la Salud.

Conflicto de intereses. Los autores declaran no tener conflicto de intereses.

Financiamiento. Este proyecto fue financiado por CONACYT: Red temática de Automanejo en Enfermedades crónicas \#970842.

Agradecimientos. Agradecemos el apoyo técnico y económico en la realización del presente estudio tanto a la Universidad Autónoma de Tamaulipas, Facultad de Enfermería Campus Tampico como al Instituto Nacional de Enfermedades Neoplásicas-Perú.

\section{REFERENCIAS}

1. Sarria-Bardales G, Limache-García A. Control del cáncer en el Perú: un abordaje integral para un problema de salud pública. Rev Peru Med Exp Salud Publica. 2013; 30(1): 93-8.

https://dx.doi.org/10.1590/s1726-46342013000100018

2. Bray F, Ferlay J, Soerjomataram I, Siegel RL, Torre LA, Jemal A. Global cancer statistics 2018: GLOBOCAN estimates of incidence and mortality worldwide for 36 cancers in 185 countries. CA Cancer J Clin. 2018; 68(6): 394-424. http://dx.doi.org/10.3322/caac.21492

3. Piñeros M, Ramos W, Antoni S, Abriata G, Medina LE, Miranda JJ, et al. Cancer patterns, trends, and transitions in Peru: A regional perspective. Lancet Oncol. 2017; 18(10): 573-86. https://doi.org/10.1016/S1470-2045(17)30377-7

4. Ramos-Muñoz WC, Guerrero-Ramírez NN. Análisis de la Situación del Cáncer en el Perú. Lima: Ministerio de Salud; 2020. https://bit.ly/3iTFCPz

5. Allemani C, Matsuda T, Di Carlo V, Harewood R, Matz M, Nikšić M, et al. Global surveillance of trends in cancer survival 2000-14 (CONCORD-3): Analysis of individual records for 37513025 patients diagnosed with one of 18 cancers from 322 population-based registries in 71 countries. Lancet. 2018; 391(10125): 1023-75. https://doi.org/10.1016/So140-6736(17)33326-3

6. Stenning-Persivale K, Savitzky-Franco MJ, Cordero-Morales A, Cruzado-Burga J, Poquioma E, Díaz-Nava E, et al. The mortality-incidence ratio as an indicator of five-year cancer survival in metropolitan Lima. Ecancermedicalscience. 2018;12:1-8. https://doi.org/10.3332/ecancer.2018.799 
7. National Cancer Institute. Cancer Statistics. Estados Unidos de América: NCI; Actualizado 2020. https://bit.ly/316R3Gi

8. Zebrack BJ. Psychological, social, and behavioral issues for young adults with cancer. Cancer. 2011; 117(S10): 2289-94. https://doi.org/10.1002/cncr.26056

9. Naughton MJ, Weaver KE. Physical and mental health among cancer survivors: Considerations for long-term care and quality of life. N C Med J. 2014; 75(4): 283-6.

https://doi.org/10.18043/ncm.75.4.283

10. Stanton AL. What happens now? Psychosocial care for cancer survivors after medical treatment completion. J Clin Oncol. 2012; 30(11): 1215-20. https://doi.org/10.1200/JCO.2011.39.7406

11. Howell D, Oliver TK, Keller-Olaman S, Davidson JR, Garland S, Samuels C, et al. Sleep disturbance in adults with cancer: A systematic review of evidence for best practices in assessment and management for clinical practice. Ann Oncol. 2014; 25(4): 791-800.

https://doi.org/10.1093/annonc/mdt506

12. Pitman A, Suleman S, Hyde N, Hodgkiss A. Depression and anxiety in patients with cancer. BMJ. 2018; 361: 1-11. https://doi.org/10.1136/bmj.k1415

13. Cuthbert CA, Farragher JF, Hemmelgarn BR, Ding Q, McKinnon GP, Cheung WY. Self-management interventions for cancer survivors: A systematic review and evaluation of intervention content and theories. Psycho-oncology. 2019; 28(11): 2119-40. https://doi.org/10.1002/pon.5215

14. Cuthbert CA, Samawi HH, Hemmelgarn BR, Cheung WY. Effectiveness and components of self-management interventions in adult cancer survivors: A protocol for a systematic review and planned meta-analysis. Syst Rev. 2018; 7(238): 1-7. https://doi.org/10.1186/s13643-018-0902-7

15. McCorkle R, Ercolano E, Lazenby M, Schulman-Green D, Schilling LS, Lorig K, et al. Self-management: Enabling and empowering patients living with cancer as a chronic illness. CA Cancer J Clin. 2011; 61(1): 50-62. https://doi.org/10.3322/caac.20093

16. Baydoun M, Barton DL, Arslanian-Engoren C. A cancer specific middle-range theory of symptom self-care management: A theory synthesis. J Adv Nurs. 2018; 74(12): 2935-46.

https://doi.org/10.1111/jan.13829

17. Organización Panamericana de la Salud. Cuidados innovadores para las condiciones crónicas: Organización y prestación de atención de alta calidad a las enfermedades crónicas no transmisibles en las Américas. Washington, DC: OPS; 2013. p. 30. https://bit.ly/3u2XP2j

18. Corbin J, Strauss A. Managing chronic illness at home: Three lines of work. Qual. Sociol. 1985; 8(3): 224-247. https://doi.org/10.1007/BFoog89485

19. Lorig KR, Holman H. Self-management education: History, definition, outcomes, and mechanisms. Ann Behav Med. 2003; 26(1): 1-7. https://doi.org/10.1207/S15324796ABM2601_01

20. Fernández-Cantón SB. La atención centrada en el paciente: una visión general sobre su enfoque en los servicios de salud. Bol. conamed OPS. 2019; 4(23): 13-23. https://bit.ly/2TFGOol

21. Audulv $\AA$, Ghahari S, Kephart G, Warner G, Packer TL. The taxonomy of everyday self-management strategies (TEDSS): A framework derived from the literature and refined using empirical data. Patient Educ Couns. 2019; 102(2):367-375. https://doi.org/10.1016/j.pec.2018.08.034

22. Cheng H, Sit JWH, Cheng KKF. A qualitative insight into self-management experience among Chinese breast cancer survivors. Psycho-oncology. 2017; 26(7): 1044-49.

https://doi.org/10.1002/pon.4279 
23. Williams AR, Mowlazadeh B, Sisler L, Williams PD. Self-reported assessment of symptoms and self-care within a cohort of U.S. veterans during outpatient care for cancer. Clin J Oncol Nurs. 2015; 19(5): 595-602. https://doi.org/10.1188/15.CJON.595-602

24. Kim SH, Kim K, Mayer DK. Self-management intervention for adult cancer survivors after treatment: A systematic review and meta-analysis. Oncol Nurs Forum. 2017; 44(6): 719-28.

https://doi.org/10.1188/17.ONF.719-728

25. Boland L, Bennett K, Connolly D. Self-management interventions for cancer survivors: a systematic review. Support Care Cancer. 2018; 26(5): 1585-95. https://doi.org/10.1007/s00520-017-3999-7

26. Heinze SB, Williams PD. Symptom alleviation and self-care among breast cancer survivors after treatment completion. Clin J Oncol Nurs. 2015;19(3):343-9. https://doi.org/10.1188/15.CJON.343-349

27. Williams PD, Lopez V, Ying CS, Piamjariyakul U, Wenru W, Hung GT, et al. Symptom monitoring and self-care practices among oncology adults in China. Cancer Nurs. 2010; 33(3): 184-93.

https://doi.org/10.1097/NCC.obo13e3181c29598

28. Schulman-Green D, Bradley EH, Knobf MT, Prigerson H, DiGiovanna MP, McCorkle R. Self-management and transitions in women with advanced breast cancer. J Pain Symptom Manage. 2011; 42(4): 517-25. https://doi.org/10.1016/j.jpainsymman.2010.12.007

29. Shneerson C, Taskila T, Holder R, Greenfield S, Tolosa I, Damery S, et al. Patterns of self-management practices undertaken by cancer survivors: Variations in demographic factors. Eur J Cancer Care (Engl). 2015; 24(5): 683-94. https://doi.org/10.1111/ecc.12252

30. Peñarrieta-de Córdova MI, Reyes G, Krederdt S, Flores F, Resendiz E, Chávez-Flores E. Automanejo en enfermedades crónicas: diabetes mellitus tipo 2, hipertensión arterial y cáncer. Rev. investig. Univ. Norbert Wiener. 2015; 4: 43-56. https://bit.ly/37lcqMd

31. Battersby MW, Ask A, Reece MM, Markwick MJ, Collins JP. The partners in health scale: The development and psychometric properties of a generic assessment scale for chronic condition self-management. Aust J Prim Health. 2003; 9(3): 41-52. https://doi.org/10.1071/PYo3022

32. Ostby PL, Armer JM, Smith K, Stewart BR. Patient perceptions of barriers to self-management of breast cancer-related lymphedema. West J Nurs Res. 2018; 40(12): 1800-17. https://doi.org/10.1177/0193945917744351

33. Geng Z, Ogbolu Y, Wang J, Hinds PS, Qian H, Yuan C. Gauging the effects of self-efficacy, social support, and coping style on self-management behaviors in chinese cancer survivors. Cancer Nurs. 2018; 41(5): 1-10. https://doi.org/10.1097/NCC.0000000000000571

34. Dunne S, Coffey L, Sharp L, Timmons A, Desmond D, Gooberman-Hill R, et al. Barriers to active self-management following treatment for head and neck cancer: Survivors' perspectives. Psycho-oncology. 2018; 27(10): 2382-8. https://doi.org/10.1002/pon.4835

35. Kidd LA. Consequences, control and appraisal: Cues and barriers to engaging in self-management among people affected by colorectal cancer-a secondary analysis of qualitative data. Health Expect. 2014; 17(4): 565-78. https://doi.org/10.1111/j.1369-7625.2012.00785.X

36. Syed IA, Nathan PC, Barr R, Rosenberg-Yunger ZR, D'Agostino NM, Klassen AF. Examining factors associated with self-management skills in teenage survivors of cancer. J Cancer Surviv. 2016; 10: 686-91. https://doi.org/10.1007/s11764-016-0514-y

37. Lorig K, Ritter PL, Turner RM, English K, Laurent DD, Greenberg J. A diabetes self-management program: 12-month outcome sustainability from a nonreinforced pragmatic trial. J Med Internet Res. 2016; 18(12): 1-11. https://doi.org/10.2196/jmir.6484 
38. Smith D, Harvey P, Lawn S, Harris M, Battersby M. Measuring chronic condition self-management in an Australian community: Factor structure of the revised partners in health (PIH) scale. Oual Life Res. 2017; 26: 149-59. https://doi.org/10.1007/s11136-016-1368-5

39. Peñarrieta-de Córdova MI, Vergel-Camacho O, Schmith-Álvarez L, Lezama-Vigo S, Rivero-Álvarez $\mathrm{R}$, Taipe-Cancho J, et al. Validación de un instrumento para evaluar el automanejo en enfermedades crónicas en el primer nivel de atención en salud. Rev. Cient. de Enferm. 2011; V(1): 15-21.

https://bit.ly/2ViYMGq

40. Lawn S, Battersby M, Harvey P, Pols R, Ackland A. A behavioural therapy approach to self-management: The Flinders program. Diabetes Voice. 2009; 54: 30-2. https://bit.ly/3BSzaB8

41. Lorig K, Stewart A, Ritter P, González V, Laurent D, Lynch J. Outcome measures for health education and other health care interventions. EE.UU.: SAGE Publications Inc; 1996.

http://dx.doi.org/10.4135/9781452232966

42. González VM, Stewart A, Ritter PL, Lorig K. Translation and validation of arthritis outcome measures into Spanish. Arthritis Rheum. 1995; 38(10): 1429-46. https://doi.org/10.1002/art.1780381010

43. Hernández-Pérez E. Relación entre percepción de salud y calidad de vida en adultos mayores con enfermedades crónicas. [Tesis]. Tampico, Tamaulipas: Universidad Autónoma de Tamaulipas; 2019.

44. Kroenke K, Strine TW, Spitzer RL, Williams JBW, Berry JT, Mokdad AH. The PHQ-8 as a measure of current depression in the general population. J Affect Disord. 2009; 114(1-3): 163-73.

https://doi.org/10.1016/j.jad.2008.06.026

45. León-Hernández RC, Peñarrieta-De Córdova MI, Gutiérrez-Gómez T. Capítulo V. Validación de instrumentos de indicadores de salud y psicosociales. Red de automanejo de enfermedades crónicas. En: Gutiérrez-Gómez T, Castañeda-Hidalgo H (Coords.). Estrategias de aprendizaje en cronicidad. México: Universidad Autónoma de Tamaulipas; 2019.

46. Lesage FX, Berjot S. Validity of occupational stress assessment using a visual analogue scale. Occup Med (Lond). 2011; 61(6): 434-6. https://doi.org/10.1093/occmed/kqro37

47. Albuja-Calo JP. Validación de la prueba de keiraku cervical para medir el estrés académico en los estudiantes de la carrera de terapia física, en el periodo octubre- diciembre del 2016 en la Pontificia Universidad Católica del Ecuador. [Tesis]. Ecuador: Pontificia Universidad Católica del Ecuador; 2018. https://bit.ly/2WqzEOu

48. Wolfe F, Michaud K, Li T. Sleep disturbance in patients with rheumatoid arthritis: Evaluation by medical outcomes study and visual analog sleep scales. J Rheumatol. 2006; 33(10): 1942-51. https://bit.ly/3zOyXgo

49. Carvajal-Valcárcel A, Martínez-García M, Centeno-Cortés C. Versión española del Edmonton Symptom Assessment Sytem (ESAS): un instrumento de referencia para la valoración sintomática del paciente con cáncer avanzado. Med. paliat. 2013; 20(4): 143-9.

https://doi.org/10.1016/j.medipa.2013.02.001

50. Lorig KR, Ritter PL, Jacquez A. Outcomes of border health spanish/english chronic disease self-management programs. Diabetes Educ. 2005; 31(3): 401-9. https://doi.org/10.1177/0145721705276574

51. Cárdenas-Castro M, Arancibia-Martini H. Potencia estadística y cálculo del tamaño del efecto en G*Power: complementos a las pruebas de significación estadística y su aplicación en psicología. Salud soc. 2014; 5(2): 210-24. https://doi.org/10.22199/S07187475.2014.0002.00006 